\title{
Pseudoscorpione von der Dominicanischen Republik (Insel Haiti)
}

von

\section{BEIER}

Mit 7 Textfiguren

\section{SUMMARY}

21 species of pseudoscorpions are recorded from the Dominican Republic, seven of them are described as new for science: Parachernes dominicanus n. sp. (l 1 of 2 q), Caribochernes pumilus n. gen. n. sp. (9 ô 13 \% 10 Nymphen), Byrsochernes caribicus n. sp. (3 +), Parazaona klapperichi n. sp. (1 ot 3 Nymphen), Chernes hispaniolicus n. sp. $(2$ o), Hesperochernes vespertilionis n. sp. (6 o 8 ㅇ 15 Nymphen), Parachelifer dominicanus n. sp. (13 ô $14+5$ Tritonymphen). The species Tyrannochthonius fastuosus Hoff and $T$. lautus Hoff are considered as junior synonyms of $T$. imitatus Hoff.

Das Naturhistorische Museum in Genf erwarb von J. und S. Klapperich eine Anzahl von Pseudoscorpionen aus der Dominikanischen Republik. Das mir zur Bearbeitung anvertraute Material enthielt die im Folgenden genannten 21 Arten, von denen 7 als neu beschrieben werden.

\section{Tyrannochthonius imitatus Hoff, 1959}

2 ô, 4 + , Cazabita, Cord. centr., 1250 m, 10.III. und 24.IV.1974.

Die Art wurde aus Jamaica beschrieben. Die vorliegenden Exemplare sind etwas kleiner als die Typenexemplare: Körper-L. ot $0,67 \mathrm{~mm}$, $+0,85-0,90 \mathrm{~mm}$; Carapax-L. o $0,24 \mathrm{~mm}$, क 0,30 mm; Palpenschere ơ $0,36 \mathrm{~mm}$, क 0,44 0,45 mm, Hand-L. o $0,12 \mathrm{~mm}$, ๆ $0,17 \mathrm{~mm}$, B. $+0,11 \mathrm{~mm}$, Finger ô $0,24 \mathrm{~mm}$, $+0,28 \mathrm{~mm}$. Das Epistom ist oft einfach gleichseitig-dreieckig.

In T. fastuosus Hoff und lautus Hoff sehe ich lediglich innerhalb der Variationsbreite der Art gelegene Populationen von imitatus. Jedenfalls reichen die von Hoff angegebenen Merkmale zur Artunterscheidung nicht aus, weshalb die beiden erstgenannten Arten in die Synonymie von imitatus fallen.

\section{Tyrannochthonius proximus Hoff, 1959}

7 ô, 12 \&, 4 Tritonymphen, Cazabita, Cord. centr., 1250 m, 24.IV. und 30.VI.1974.

Bisher ebenfalls nur von Jamaica bekannt. Das bisher unbeschriebene Männchen hat eine Körperlänge von $1 \mathrm{~mm}$, der Carapax ist 0,35, die Palpenschere 0,60 mm lang. 
Die Interkalarzähne (Mikrodentikel) der Palpenfinger sind zu kleinen, oft nur schwer sichtbaren Körnchen reduziert.

Lechytia trinitatis Beier, 1970

1 q (Torso), Boca Chica, 10 m, 13.XII.1970.

Die durchgehend bezahnten Palpenfinger sprechen für die Zugehörigkeit zu dieser Art.

\section{Ideobisium crassimanum Balzan, 1891}

2 ô, 5 q, 12 Nymphen aller Stadien, Cazabita, Cord. centr., 1250 m, 8.VII.1972, 10.III., 24.IV. und 30.VI.1974.

Die Art ist über das nördlichste Südamerika und Zentralamerika verbreitet.

Olpiolum monae Hoff, nom. nov. 1964

pro Pachyolpium medium Hoff 1945.

1 ô, 1 ㅇ, Bani, 65 m, 4.III.1972.

Höchstwahrscheinlich gehören auch eine Trito- und eine Protonymphe von gleichen Fundort, 23.I. und 22.X.1972 dieser von der Insel Mona und von Jamaica bekannten Art an.

\section{Planctolpium arboreum Hoff, 1964}

1 , , Boca Chica, $10 \mathrm{~m}, 27$. III.1971.

Diese zu den Garypidae gehörende Art wurde von Jamaica beschrieben.

Garyops depressa Banks, 1909

8 oิ, 15 ㅇ, Bani, 65 m, 20.II.1971, 28.V.-30.IX.1972.

War bisher nur von Florida bekannt.

Paratemnus elongatus (Banks, 1895)

syn. Atemnus floridanus Tullgren, 1900.

1 ô, 3 ค, Boca Chica, 10 m, 5.XII.1970-19.VI.1971.

Wahrscheinlich nur eine Form von P. nidificator (Balzan, 1890). (Siehe zu dieser Art auch Hoff, 1946, Amer. Mus. Novit. 1318, p. 3 und Hoff, 1964, ibid. 2198, p. 2.)

Lustrochernes communis (Balzan, 1890)

17 ô, 12 ㅇ, 15 Tritonymphen, Boca Chica, 10 m, 21.XI. und 13.XII.1970, 22.V.-20.XI.1971; Bani, 65 m, 20.II. und 16.VIII. 1971; Colonia Ramfis, Cord. centr., $1000 \mathrm{~m}, 1 . \mathrm{V} .1974$.

Die Art ist in Südamerika weit verbreitet und kommt auch auf Trinidad vor. (Siehe auch Hoff, 1946, Amer. Mus. Novit. 1318, p. 4, mit Literaturhinweisen.) 


\section{Parachernes dominicanus n. sp. (Fig. 1)}

Holotype : : Boca Chica, 10 m, 5.VI.1971 (Mus. Genf).

Paratypen: 1 우 vom gleichen Fundort, 22.V.1971; 1 ô, Haiti, Kenskoff Pass, ca. 2000 m, 13.XII.1972 (beide Mus. Genf).

Carapax laterobasal und die drei vordersten Tergite lateral mit hellen Makeln, die vom 1. bis zum 3. Tergit allmählich kleiner werden. Integument relativ grob und nicht sehr dicht granuliert, die Granulation oral auf dem Carapax, auf dem letzten



FIG. 1.

Parachernes dominicanus n. sp., linke Palpe des Männchens, rechte des Weibchens und Schere des Weibchens laterodorsal.

Abdominaltergit sowie lateral auf Femur und Tibia der Palpen und auf der ganzen Hand erloschen. Vestituralborsten steif und ziemlich lang, auf der Dorsalseite des Körpers fast stiftförmig, relativ dick, stumpf, distal ziemlich derb gezähnt, auf den Palpen größtenteils mit Medialzähnchen und Endgabel; Medialborsten der Hand beim Männchen stark verlängert, nur mit feiner Endgabel. - Carapax so lang wie hinten breit, seine vordere Querfurche ziemlich tief, die dem Hinterrand genäherte subbasale flach; Hinterrand mit 6 Borsten. Keine Augenflecke. Halbtergite mit je 4 bis 5 Hinterrandborsten. Endtergit mit 4 Discalborsten und lateralen Tastborsten. Chelicerenstamm 
mit 5 Borsten, $B$ und $S B$ gezähnt. Galea gedrungen, beim Weibchen mit 6 kurzen Seitenästchen in den beiden proximalen Dritteln. Galealborste bis zum Ende der Galea reichend. Palpen gedrungen. Trochanterhöcker kaum ausgeprägt. Femur abrupt gestielt, ebenso wie die Tibia 2,2 bis 2,3 mal länger als breit; Hand 1,7 mal länger als breit und 1,5 mal länger als dick; Schere mit Stiel 2,4 bis 2,7 mal, ohne Stiel 2,2 bis 2,4 mal länger als dick. Finger so lang wie oder etwas länger als die Hand ohne Stiel und etwas länger als deren Dicke, lateral je mit 6 bis 7, medial mit 2 Nebenzähnen. Die 4 medialen Tasthaare eine Vierergruppe an der Basis des festen Fingers bildend, it unmittelbar bei ist stehend; st des beweglichen Fingers halbwegs zwischen $t$ und $s b, t$ in der Fingermitte befindlich. Beine ziemlich gedrungen, die Tastborste am Hintertarsus distal der Gliedmitte stehend. Weibliches Genitalfeld mit einer annähernd dreieckigen Gruppe von etwa 22 sehr dicht gestellten Börstchen. - Körper-L. ô 1,5, † $1,8 \mathrm{~mm}$; Carapax-L. ơ $0,65 \mathrm{~mm}$, † $0,72 \mathrm{~mm}$, B. of $0,60 \mathrm{~mm}$, $+0,72 \mathrm{~mm}$; Palpen: ô Femur-L. 0,52 mm, B. 0,22 mm, Tibia-L. 0,54 mm, B. 0,24 mm, Hand-L. 0,52 mm, Dicke $0,35 \mathrm{~mm}$, Finger-L. $0,46 \mathrm{~mm}$; + Femur-L. 0,54 mm, B. 0,25 mm, Tibia-L. $0,54 \mathrm{~mm}$, B. $0,25 \mathrm{~mm}$, Hand-L. 0,65 mm, B. $0,37 \mathrm{~mm}$, Dicke $0,45 \mathrm{~mm}$, Finger-L. $0,49 \mathrm{~mm}$.

Die neue Art gehört in die Verwandtschaft von $P$. distinctus Beier und tumimanus Hoff, hat jedoch längere, nicht gekeulte, sondern stiftförmige und gezähnte Vestituralborsten.

\section{Parachernes (Scapanochernes) compressus (Tullgren, 1907)}

3 ô, 4 \&, Colonia, Cord. centr., 1000 m, 21.IV.-21.VI.1972; 1 ô, 1 +, 1 Tritonymphe, Constanza, 1250 m, 14.VI.1972; 1 ô, St. Domingo, 30 m, 17.XI.1970.

Typische Färbung des Männchens: Metazone des Carapax bis auf einen basalen Mittelfleck hell, Halbtergite des 1. bis 3. Segments in den beiden lateralen Dritteln weiß, im medianen Drittel braun, die übrigen größtenteils braun, nur lateral breit aufgehellt, doch können die vorderen Tergite auch weitgehend braun sein. Beim Weibchen ist diese Färbung schwächer ausgeprägt. Die Art ist in der Größe sehr variabel. Die Maße der kleinsten hier vorliegenden Männchen betragen: Körper-L. 2,2 mm; Carapax-L. 0,75 mm, B. 0,68 mm; Palpen: Femur-L. 0,62 mm, B. 0,25 mm, Tibia-L. 0,62 mm, B. 0,30 mm, Hand-L. 0,70 mm, B. 0,53 mm, Finger-L. 0,56 mm. Die Maße der kleinsten Weibchen sind ähnlich: Carapax-L. 0,75 mm, B. 0,77 mm; Palpenfemur-L. $0,63 \mathrm{~mm}$, B. $0,28 \mathrm{~mm}$, Tibia-L. 0,62 mm, B. 0,28 mm, Hand-L. 0,75 mm, B. 0,50 mm, Finger-L. $0,60 \mathrm{~mm}$. Keines der Exemplare erreicht die von Tullgren angegebenen Maße. - Wahrscheinlich ist Chelanops diversus (Banks, 1909) mit dieser Art synonym.

\section{Caribochernes n. gen.}

Sehr klein. Integument dicht und sehr grob granuliert. Vestituralborsten zum Großteil stark gekeult, ziemlich kurz. Carapax etwa 1/5 länger als breit, mit Augenflecken, die subbasale Querfurche dem Hinterrand stark genähert. Tergite geteilt, einreihig beborstet, Endtergit ohne Tastborsten. Intersegmental- und Pleuralmembran dicht hispid granuliert. Chelicerenstamm mit 5 Borsten, $S B$ apikal fein gegabelt. Flagellum mit 3 Borsten. Palpen gedrungen, Femur abrupt gestielt. Nebenzähne spärlich und auf den Apikalteil der Finger beschränkt. Die 4 medialen Tasthaare des festen Fingers in einer Vierergruppe nahe der Fingerbasis stehend, est ebenfalls weit proximal befindlich; $t$ des beweglichen Fingers an st genähert und proximal der Fingermitte 
stehend. Beine ziemlich gedrungen, der Hintertarsus mit einer langen apikalen Tastborste, die unmittelbar über den beiden Endborsten am Vordereck steht und mit diesen eine Dreiergruppe bildet. Klauen einfach. Vorderes Genitaloperculum des Männchens groß und breit, kaudal leicht sinuiert, zur Gänze mäßig dicht beborstet. Weibliches Genitalfeld mit kleinem Borstenfeld.

Genustypus: Caribochernes pumilus n. sp.

Vorkommen: Große Antillen (Ins. Haiti).

Die neue Gattung stimmt in der Stellung der Tasthaare der Palpenfinger mit Parachernes überein, unterscheidet sich jedoch von diesem durch die an das Gliedende gerückte Tastborste des Hintertarsus, die stark gekeulten Vestituralborsten, das Fehlen von Tastborsten auf dem Endtergit und die für einen Chernetiden ungewöhnlich geringe Körpergröße.

\section{Caribochernes pumilus n. sp. (Fig. 2)}

Holotype $\hat{o}$ und Allotype + : Rep. Dominica, Colonia, Cord. centr., $1000 \mathrm{~m}$, 4.IV.1972 (Mus. Genf).

Paratypen: 8 ô, 12 \%, 8 Deuto- und Tritonymphen, Colonia Ramfis, Cord. centr., 1000 m, 26.II., 18.III., 1. und 6.V., 10. und 21.VI., 12.VIII.1972, 21.I. und 1.V.1974; 1 \&, 2 Tritonymphen, Cazabita, Cord. centr., 1250 m, 30.VI.1974.

Sehr kleine, einfarbig braune Tiere. Integument sehr grob granuliert, der Zwischenraum zwischen den halbkugeligen Granula höchstens so groß wie ein Korndurchmesser, meist aber kleiner. Vestituralborsten kurz, auf dem Dorsum, auf der Medial- und

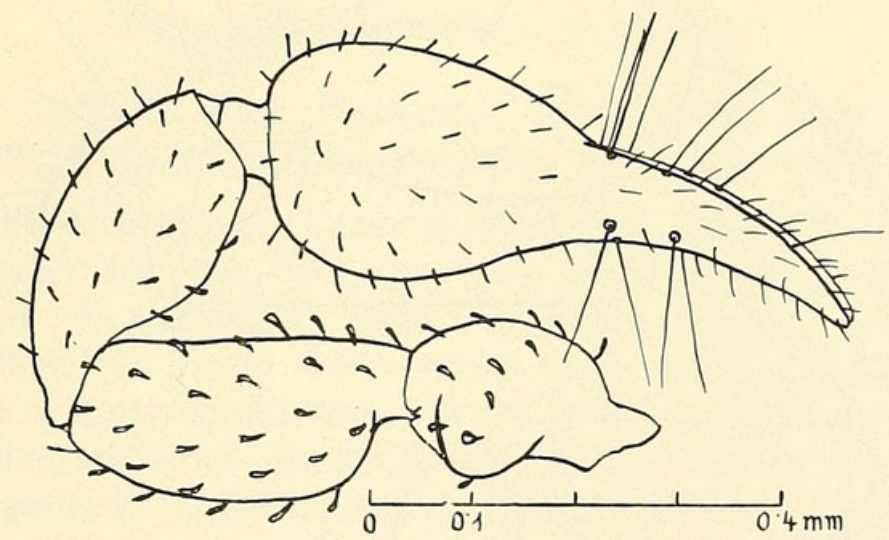

FIG. 2.

Caribochernes pumilus n. gen. n. sp.,, , linke Palpe.

Dorsalseite der proximalen Palpenglieder sowie auf der Dorsalseite der Mittel- und Hinterbeine stark gekeult und längsgerieft, apikal breit verrundet, die übrigen fein und spärlich gezähnt; Ventralborsten der Beine etwas länger und einfach. - Carapax nur wenig länger als breit, in der Mesozone am gröbsten granuliert, seine beiden Querfurchen mäßig tief, die subbasale von der vorderen Furche etwa doppelt so weit entfernt wie vom Hinterrand, dieser mit 12 bis 14 Borsten. Augenflecke meist gut entwickelt. Halbtergite größtenteils mit je 7 bis 9 Borsten, davon eine auf den hinteren Segmenten in subdiscaler Stellung. Endtergit mit zwei Discalborsten, die lateralen Randborsten etwas länger, aber ebenfalls stark gekeult. Sternitborsten kurz und einfach. Endsternit 
mit zwei kurzen lateralen Tastborsten. Galea des Weibchens mit 6 kurzen Ästchen in den beiden distalen Dritteln, die des Männchens nur mit wenigen kurzen Zähnchen. Palpenfemur 2,1 mal, Tibia 2,2 mal, Hand 1,6 mal, Schere mit Stiel 2,8 mal, ohne Stiel 2,6 mal länger als breit. Finger ein wenig länger als die Hand ohne Stiel, nur lateroapikal mit Nebenzähnen, und zwar der feste mit 4, der bewegliche mit 2. Stellung der Tasthaare wie in der Genusdiagnose. Die Tastborste am Hintertarsus ein wenig länger als die beiden Endborsten, mit denen sie eine Gruppe bildet. Borstenfeld des weiblichen Genitalfeldes mit etwa 14 kurzen Börstchen, die beiden folgenden Segmente mit je einer Querreihe von 8 Borsten. - Körper-L. ơ 0 7 95-1,10 mm; Carapax-L. 0,490,50 mm, B. 0,40-0,42 mm; Palpen: Femur-L. 0,33 mm, B. 0,16 mm, Tibia-L. 0,35 mm, B. 0,16 mm, Hand-L. 0,35 mm, B. 0,23 mm, Finger-L. 0,32 mm.

\section{Byrsochernes caribicus n. sp. (Fig. 3)}

Holotype + : Rep. Dominica, Boca Chica, 10 m, 13.XII.1970 (Mus. Genf).

Paratypen: 1 q vom gleichen Fundort, 20.XI.1971; 1 \%, Bani, 65 m, 20.II.1971.

Carapax und Tergite ziemlich blaß rötlichbraun, ersterer basal etwas aufgehellt, Palpen dunkler rotbraun. Carapax ein wenig länger als breit, fein und nicht sehr scharf granuliert, ohne Augenflecke, seine Querfurchen fast gänzlich eingeebnet, nur seitlich

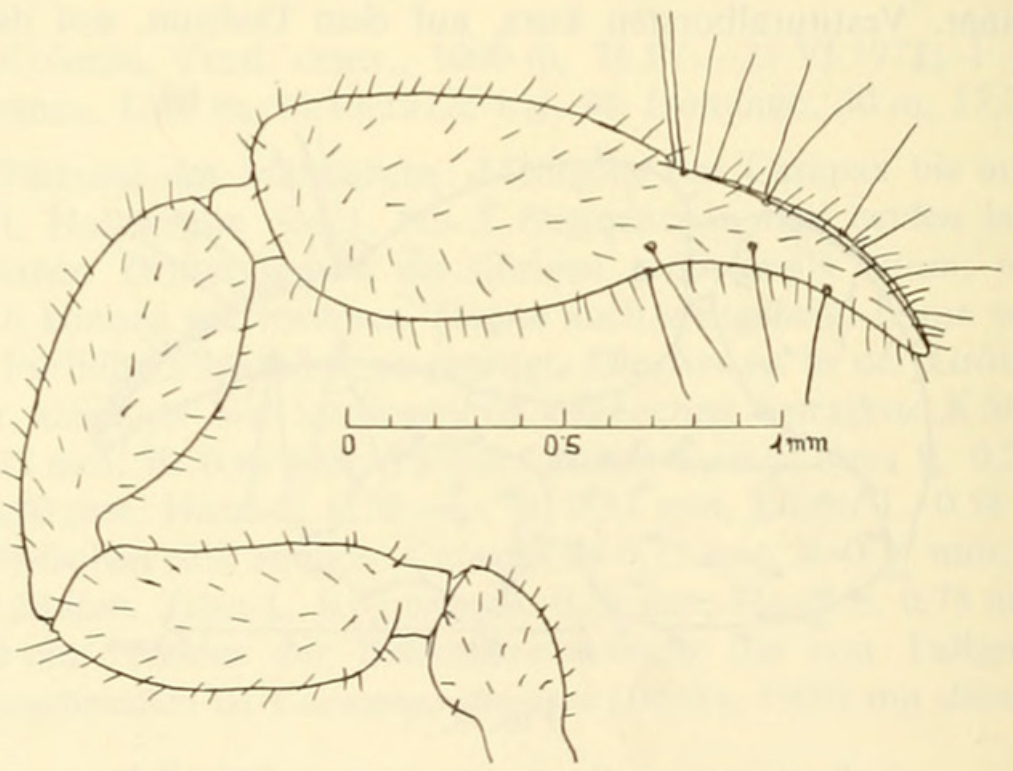

FIG. 3.

Byrsochernes caribicus n. sp., ㅇ, linke Palpe.

erkennbar, die subbasale dem Hinterrand genähert. Tergite zart retikuliert, mit Ausnahme des letzten geteilt. Die Halbtergite größtenteils mit je 6 Hinterrandborsten, diese steif, terminal stumpf und gezähnt, auf den vorderen Segmenten kurz, auf den hinteren an Länge zunehmend; Endtergit mit etwa 12 Randborsten und 4 Discalborsten, die lateralen Rand- und Discalborsten sehr stark verlängert (Tastborsten). Pleuralmembran und Intersegmentalmembranen fein und sehr dicht längsgestreift. Sternitborsten spitzig; Endsternit mit lateralen Tastborsten. Chelicerenstamm mit 
5 Borsten, $B$ und $S B$ fein und spärlich gezähnt, $E S$ ziemlich lang. Flagellum mit 3 Borsten, die 3. fein und kurz gesägt. Serrula mit 25 Lamellen. Galea des Weibchens lang, mit zarter Terminalgabel und davor im Apikalteil mit 4 sehr kurzen Ästchen, von denen die drei ventrolateralen hintereinander stehen. Palpen mit Ausnahme der Hand dicht und ziemlich fein granuliert, ihre Vestituralborsten relativ kurz, steif, distal und apikal gezähnt, die der Hand am längsten. Trochanterhöcker stark verflacht. Femur abrupt gestielt, dann von gleicher Breite, 2,7 mal länger als breit; Tibia kurz gestielt, 2,3 bis 2,5 mal, Hand 2 mal, Schere mit Stiel 3,2 mal, ohne Stiel 3 mal länger als breit. Finger viel kürzer als die Hand und nicht ganz um die Hälfte länger als deren Breite, mit zahlreichen Nebenzähnen. Das Tasthaar ist des festen Fingers gegenüber von est und fast halbwegs zwischen $i s b$ und it, st des beweglichen Fingers an $s b$ genähert. Tibia des 4. Beinpaares ohne Tastborste, Tarsus mit einer sehr langen Tastborste am Beginne des 2. Glieddrittels. Klauen plump, einfach. - Körper-L. o $4 \mathrm{~mm}$; Carapax-L. 1,10 mm, B. $0,95 \mathrm{~mm}$; Palpen: Femur-L. 0,90-0,95 mm, B. 0,33-0,35 mm, Tibia-L. 1,02$1,03 \mathrm{~mm}$, B. $0,41-0,45 \mathrm{~mm}$, Hand-L. $1,05 \mathrm{~mm}$, B. $0,52-0,55 \mathrm{~mm}$, Finger-L. $0,75-$ $0,80 \mathrm{~mm}$.

Von B. ecuadoricus m., 1959, vor allem durch das nicht so stark an $s b$ genäherte Tasthaar st des beweglichen Palpenfingers, kürzere und weiter distal stehende Ästchen der Galea, geringere Zahl der Serrulalamellen, gedrungenere Palpentibia und geringere Größe unterschieden.

Die Gattung Byrsochernes gehört nicht zu den Lamprochernetinae, sondern zu den Chernetinae (Chernetini), was das Fehlen einer Tastborste auf den Hintertibien beweist, und ist in die Nähe von Parazaona zu stellen.

Parazaona klapperichi n. sp. (Fig. 4)

Holotype ô: $^{-}$südliche Rep. Dominica, Sabaneta, in einer Fledermaushöhle, 13.III.1974. - Außerdem liegen 1 Deutonymphe und 2 Protonymphen vor.

Hartteile dunkel rötlichbraun. Vestituralborsten kurz, ziemlich derb, distal gezähnt. Carapax etwas länger als breit, sehr dicht und ziemlich fein granuliert, seine Querfurchen undeutlich, die subbasale dem Hinterrand genähert. Augenflecke fehlen. Tergite mit Ausnahme des 1. und letzten geteilt, dicht schuppig skulpturiert. Die Halbtergite mit je 6 bis 8 Hinterrandborsten, die der mittleren Segmente auch mit 1 Seitenrand- und 1 Medianrandborste. Endtergit mit lateralen Tastborsten. Pleuralmembran kurz und hispid gestreift. Halbsternite mit etwa 10 einfachen Randborsten, die der hinteren Segmente mit einer dem Medianrand genäherten, verlängerten Discalborste. Chelicerenstamm mit 5 Borsten, $B$ apikal kurz gegabelt, $E S$ länger als $B$. Serrula mit 23 Lamellen. Flagellum mit 3 Borsten, die distale einseitig in Abständen gesägt. Galea mit kurzer Endgabel, davor mit 3 Ästchen hintereinander, das distale am längsten. Palpen einschließlich der Hand dicht und ziemlich fein granuliert, mit kurzen Vestituralborsten, die medialen Borsten gezähnt, die lateralen etwas länger und größtenteils einfach zugespitzt. Dorsaler Trochanterhöcker gerundet, ventraler flach. Femur kurz, aber nicht abrupt gestielt, wie die Tibia 3,4 mal, Hand 2 mal, Schere mit Stiel 3,5 mal, ohne Stiel 3,2 mal länger als breit. Finger nicht ganz so lang wie die Hand ohne Stiel, die Nebenzähne lateral gut entwickelt und zahlreich, medial reduziert. Das Tasthaar ist proximal von dem fast in der Fingermitte stehenden est, dieses näher bei et als bei est, it distal bei et; st des beweglichen Fingers ein wenig näher bei $s b$ als bei $t$. Hintertarsus mit einer sehr langen Tastbortse in der Mitte des Gliedes. Krallen ziemlich schlank, aber kräftig. - Körper-L. ô 4,5 mm; Carapax-L. 1,20 mm, B. $1 \mathrm{~mm}$; Palpen: 
Femur-L. $1,30 \mathrm{~mm}$, B. 0,38 mm, Tibia-L. $1,45 \mathrm{~mm}$, B. 0,43 mm, Hand-L. $1,30 \mathrm{~mm}$, B. $0,62 \mathrm{~mm}$, Finger-L. $1,03 \mathrm{~mm}$.

Von der nahe verwandten $P$. bocki (Tullgren) aus Bolivien und Peru durch bedeutendere Größe, undeutliche Querfurchen des Carapax, schlankere Palpentibia des Männchens und proximal von est stehendes Tasthaar ist des festen Palpenfingers unterschieden.

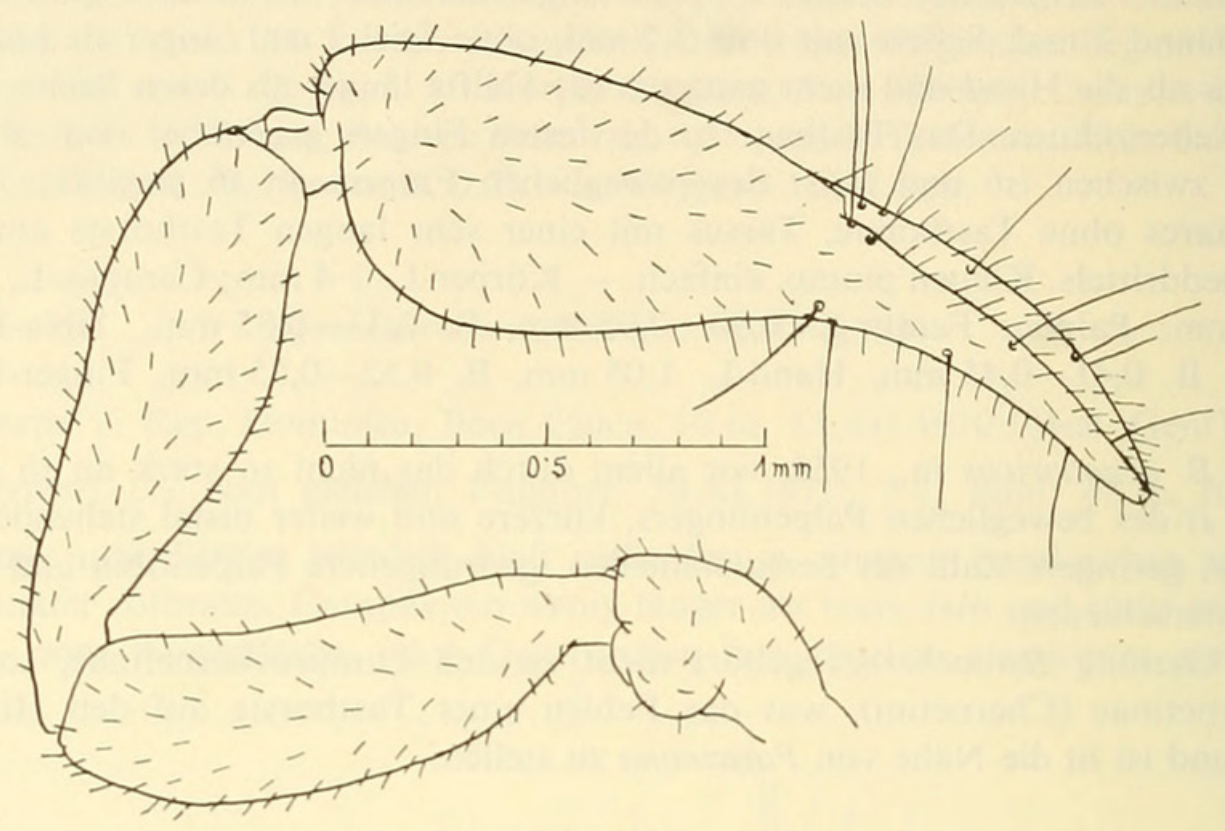

FIG. 4.

Parazaona klapperichi n. sp., ô, linke Palpe.

Chernes hispaniolicus n. sp. (Fig. 5)

Holotypus ơ: Dominicanische Republik, San Cristobal, in einer Fledermaushöhle, 29.VII.1974 (Mus. Genf).

Paratype: 1 ô vom gleichen Fundort (Mus. Wien).

Carapax und Palpen ziemlich dunkel rötlichbraun, Tergite stumpf bräunlich. Vestituralborsten durchwegs deutlich gekeult, nur diejenigen auf der Lateralseite von Tibia und Hand der Palpen sowie auf den Sterniten und auf der Ventralseite der Beine bloß gezähnt. - Carapax kaum länger als breit, sehr dicht und mäßig grob körnig granuliert, ohne Augenflecke, seine beiden Querfurchen ziemlich breit und tief, scharf gerandet, die subbasale dem Hinterrand stark genähert. Tergite mit Ausnahme des 11. breit geteilt, dicht körnig granuliert. Halbtergite der mittleren Segmente mit je 7 Hinterrandborsten sowie 1 Seiten- und Medianrandborste, ohne Discalborsten; alle Borsten ziemlich stark gekeult, auch auf den hinteren Segmenten kurz. Endtergit mit 8 Randborsten und 2 Discalborsten, ohne Tastborsten. Die hinteren Sternite fein, aber deutlich granuliert. Vorderes Genitaloperculum mit 24 Borsten, davon 4 verlängert. Chelicerenstamm mit 5 Borsten, $B$ und $S B$ gezähnt, ES sehr kurz. Flagellum mit 3 Borsten, die basale schmal und kaum halb so lang wie die 2., diese spärlich gezähnt, die 3. in der Distalhälfte einreihig dicht gefiedert. Serrula mit 15 Lamellen. Galea des Männchens kurz, mit kurzer Terminalgabel und zwei kurzen Ästchen in den beiden distalen Dritteln. Palpen dicht und mäßig grob granuliert. Coxen oral granuliert. Tro- 
chanterhöcker rund. Femur abrupt gestielt, distad leicht verjüngt, 2,6 mal, Tibia 2,2 mal, Hand 1,6 mal, Schere mit Stiel 3 mal, ohne Stiel 2,6 mal länger als breit. Hand distalwärts ziemlich stark verschmälert. Finger fast gerade, nicht ganz so lang wie die Hand mit Stiel, distal mit Nebenzähnen, die lateral etwas zahlreicher sind als medial. Das Tasthaar est an esb genähert, ist distal von est und nahe bei it; st des beweglichen Fingers an $t$ genähert. Hintercoxen des Männchens spärlich und ziemlich kurz behaart.

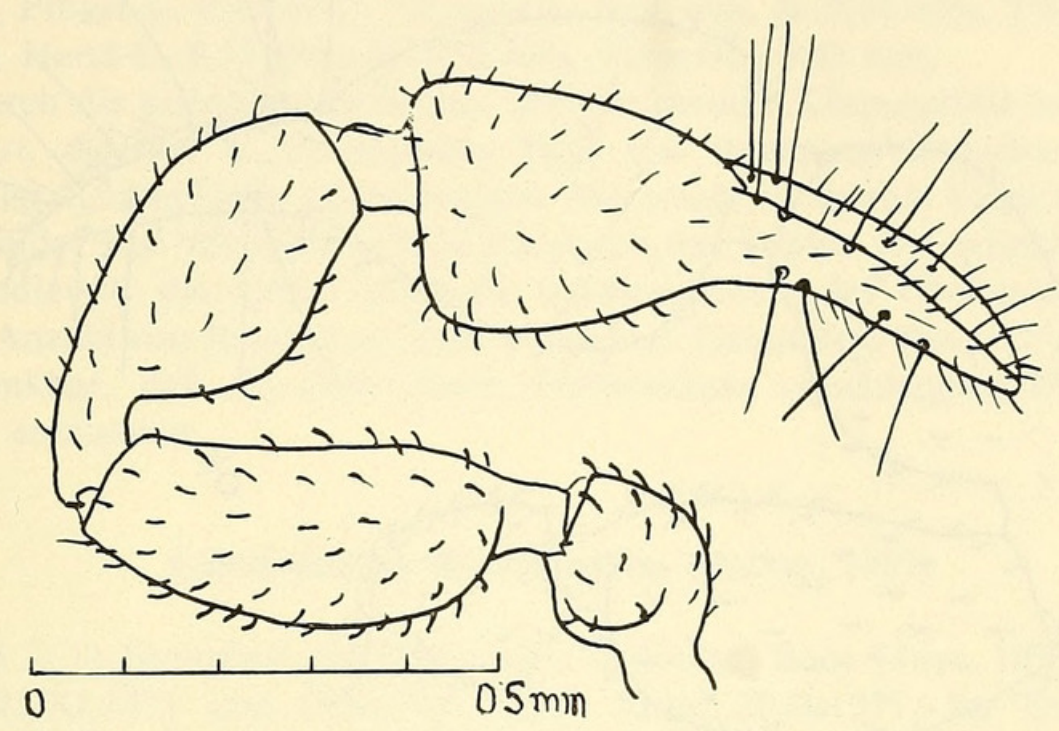

FIG. 5.

Chernes hispaniolicus n. sp., ô, linke Palpe.

Hintertarsus ohne Tastborste. - Körper-L. ô $1,5 \mathrm{~mm}$; Carapax-L. 0,60 mm, B. $0,55 \mathrm{~mm}$; Palpen: Femur-L. 0,51 mm, B. 0,20 mm, Tibia-L. 0,48 mm, B. 0,22 mm, Hand-L. 0,43 mm, B. 0,26 mm, Finger-L. 0,40 mm.

Die erste neotropische Art dieser bisher nur aus der Paläarktis bekannten Gattung, die sich durch ihre geringe Größe und die schwach gebauchte, distalwärts stark verengte Palpenhand auszeichnet. Zeigt habituelle Ähnlichkeit mit dem nordamerikanischen Reginachernes ewingi Hoff, 1949, doch hat dieser 4 Flagellumborsten.

\section{Hesperochernes vespertilionis n. sp. (Fig. 6)}

Holotypus ô: südl. Dominicanische Republik, Sabatena, 13.III.1974, in einer Fledermaushöhle (Mus. Genf).

Paratypen: 5 ô, 8 ค, 15 Nymphen aller Stadien vom gleichen Fundort, 13.III.1974 (Mus. Genf und Wien).

Carapax und Palpen hell rotbraun, Tergite gelblichbraun. Integument dicht und mäßig grob granuliert. Vestituralborsten kurz, größtenteils derb, gezähnt und leicht bis deutlich gekeult, nur auf der Lateralseite der Palpenglieder zart. - Carapax etwas länger als breit, mit breiten und ziemlich tiefen Querfurchen, die subbasale dem Hinterrand 2/3 näher gelegen als der vorderen Furche; Hinter rand meist mit 14 Borsten, von denen gewöhnlich 2 in subdiscaler Stellung stehen. Augenflecke fehlen. Halbtergite größtenteils mit 9 gekeulten Hinterrandborsten, die der hinteren Segmente mit 7 bis 8, 
von denen sich 1 bis 2 in subdiscaler Stellung befinden, vom 4. Segment an auch eine Seitenrandborste. Endtergit ungeteilt, mit 2 Discalborsten, ohne Tastborsten. Sternitborsten nur auf den hinteren Segmenten leicht gekeult, Endsternit ohne Tastborsten. Pleuralmembran hispid gestreift. Chelicerenstamm normal mit 5 Borsten, $S B$ kräftig

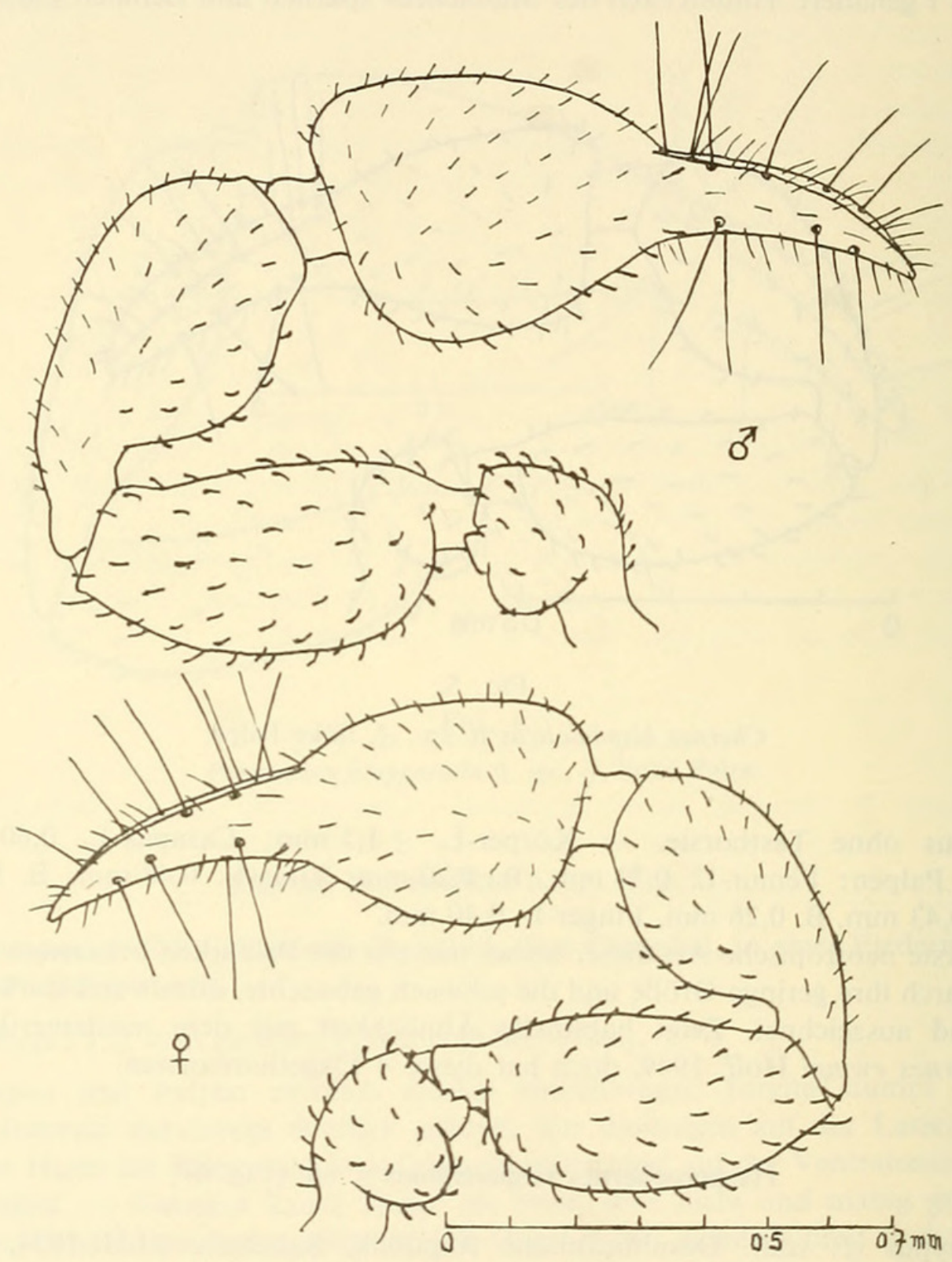

FIG. 6 .

Hesperochernes vespertilionis n. sp., linke Palpe $\widehat{0}$, rechte $q$.

und als einzige apikal gezähnt, ES kurz. Flagellum mit 4 Borsten, die distale breit und einseitig grob gesägt, die zwei basalen kurz. Serrula mit 18 Lamellen. Galea in der Distalhälfte mit 5 Ästchen, die beim Weibchen etwas länger sind als beim Männchen. Palpen ziemlich gedrungen. Trochanterhöcker gut entwickelt. Femur abrupt gestielt, 2,2 mal, Tibia 2,1 mal ( $(\hat{)})$ bis 2,2 mal ( $($ ), Hand 1,5 mal, Schere mit Stiel 2,5 mal ( $\hat{o})$ bis $2,8 \mathrm{mal}(+)$, ohne Stiel $2,3 \mathrm{mal}(\hat{\jmath})$ bis $2,6 \mathrm{mal}(\uparrow)$ länger als breit. Finger so lang 
wie die Hand ohne Stiel () oder etwas kürzer ( $\left(^{\circledR}\right)$, mit Nebenzähnen; das Tasthaar ist des festen Fingers ziemlich weit distal von est und nahe bei it, est an esb genähert, $s t$ des beweglichen Fingers viel näher bei $t$ als bei $s b$. Beine schlank, Hintertarsus ohne Tastborste. Genitaloperculum des Männchens kurz beborstet. Genitalregion des Weibchens mit etwa 22 kleinen Börstchen. - Körper-L. ô 2-2,1 mm, ㅇ 2,3-2,5 mm; Carapax-L. ot $0,74 \mathrm{~mm}$, क $0,73-0,79 \mathrm{~mm}$, B. ot $0,63 \mathrm{~mm}$, f $0,64-0,66 \mathrm{~mm}$; Palpen: ô Femur-L. 0,63 mm, B. 0,29 mm, Tibia-L. 0,66 mm, B. 0,32 mm, Hand-L. 0,61 mm, B. $0,41 \mathrm{~mm}$, Finger-L. $0,46 \mathrm{~mm}$; क Femur-L. 0,61 mm, B. 0,28 mm, Tibia-L. $0,60 \mathrm{~mm}$, B. $0,27 \mathrm{~mm}$, Hand-L. $0,53 \mathrm{~mm}$, B. $0,35 \mathrm{~mm}$, Finger-L. $0,45 \mathrm{~mm}$.

Eine durch die gedrungenen Palpen und die geringe Körpergröße sehr gut gekennzeichnete Art, die mit $H$. crassopalpus Hoff von Arkansas nächstverwandt ist und bezüglich aller Körpermaße und sonstigen Merkmale in dessen Variationsbreite fällt. Sie unterscheidet sich von diesem lediglich durch das Fehlen von lateralen Tastborsten auf dem Endtergit, die apikal gezähnte Subbasalborste des Chelicerenstammes und die größere Anzahl von Borsten auf dem weiblichen Genitalfeld (etwa 22 gegen rund 14). Es wäre denkbar, daß sie einer durch Fledermäuse verschleppten Population von crassopalpus entstammt.

\section{Cacodemonius serratidentatus (Balzan, 1891)}

33 ô, 45 †, 11 Nymphen von folgenden Fundorten: Boca Chica, 10 m, 23.XII.1970, 27.III. bis 21.XI.1971 und 16.I.1972; Bani, 65 m, 20.II.1971; St. Domingo, $30 \mathrm{~m}$, 13.III.1971; Medina bei San Cristobal, 17.IV.1971; Cacao, Cord. centr., 29.II.1972; Cazabita, Cord. centr., 1250 m, 31.X.1971 und 28.XI.1971; La Vega, Cibao, 16.IX.1973; Colonia Ramfis, Cord. centr., 1000 m, 10. bis 18.III.1972 und 21.I.1974.

In der neotropischen Region weit verbreitet.

\section{Dolichowithius simplex Beier, 1932}

\section{2 , 1 Protonymphe, Colonia Ramfis, Cord. centr., 1000 m, 1.V.1974.}

Die beiden Weibchen gehören höchstwahrscheinlich dieser bisher nur im männlichen Geschlecht von Portorico bekannten Art an. Ihre Maße betragen: Körper-L. $3 \mathrm{~mm}$; Carapax-L. 0,68 mm, B. 0,55 mm; Palpen: Femur-L. 0,55 mm, B. 0,16 mm, Tibia-L. $0,55 \mathrm{~mm}$, B. $0,17 \mathrm{~mm}$, Hand-L. $0,55 \mathrm{~mm}$, B. $0,28 \mathrm{~mm}$, Finger-L. $0,30 \mathrm{~mm}$. Das Femur ist also 3,4 mal, die Tibia 3,2 mal, die Hand $2 \mathrm{mal}$, die Schere mit Stiel 3 mal, ohne Stiel 2,7 mal länger als breit. Die Vestituralborsten sind, wie beim Männchen, nur sehr schwach gekeult. Die Halbtergite tragen 5 bis 6 Hinterrandborsten, von denen auf den hinteren Segmenten eine in discaler Stellung steht, und außerdem je eine Seiten- und Medianrandborste. Nur das Endsternit mit lateralen Tastborsten.

\section{Parachelifer dominicanus n. sp. (Fig. 7)}

Holotype $\sigma^{\wedge}$ und Allotype क: Dominicanische Rep., Boca Chica, 10 m, 19.VI.1971 (Mus. Genf).

Paratypen: $12 \hat{\jmath}, 13$, 5 Tritonymphen von folgenden Fundorten: Boca Chica, $10 \mathrm{~m}$, 5. und 13.XII.1970, 7.VIII., 6. und 20.XI.1971, 25.III.1972; Bani, 65 m, 20.II. 
und 16.VIII.1971, 23.I.1973; St. Domingo, 30 m, 24.XII.1970; Limon de Juna, 8.VI.1971; Colonia, Cord. centr., 1000 m, 1.V.1972; Constanza, Cord. centr., 1250 m, 14.VI.1972.

Integument dicht und ziemlich grob granuliert, der Carapax und die Medialseite von Femur und Tibia der Palpen mit mehreren gröberen Körnern, an deren Basis je

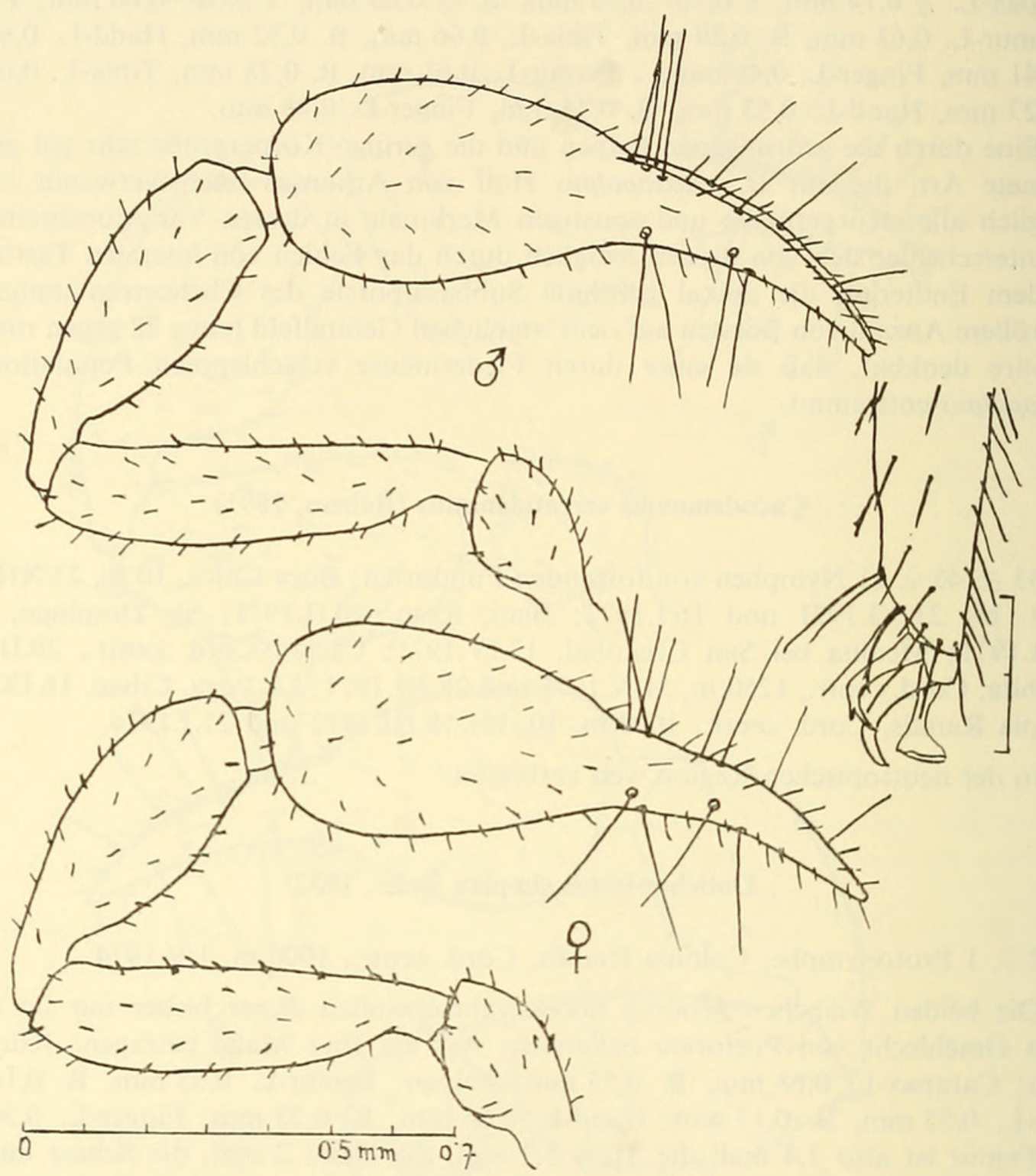

FIG. 7.

Parachelifer dominicanus n. sp., linke Palpe von $\widehat{o}$ und + , Ende des männlichen Vordertarsus.

eine Borste entspringt. Vestituralborsten kurz, stumpf, gezähnt, die Tergalborsten nur sehr schwach gekeult. - Carapax etwas länger als breit, seine Querfurchen schmal, aber ziemlich tief, die subbasale von der vorderen Furche doppelt so weit entfernt wie vom Hinterrand. Augen mit flacher Linse. Hinterecken des Carapax beim Männchen mit einem kurzen, stumpfen Dornfortsatz. Tergite nur schmal und undeutlich geteilt, das letzte und beim Männchen auch das 1. ungeteilt; beim Männchen die 4 vorderen Tergite gut sklerotisiert und dunkelbraun, die 3 folgenden bis auf die Seitenränder, die Tergite 7 bis 11 gänzlich desklerotisiert und weißlich; beim Weibchen alle Tergite 
schwach sklerotisiert und aufgehellt. Tergite 1 bis 6 des Männchens mit kräftigen Seitenrandleisten, die sich nach hinten in einen allmählich kürzer werdenden Dorn fortsetzen, Tergite 7 und 8 mit schwacher, unbewehrter Randleiste. Halbtergite mit 4 (selten 5) Hinterrandborsten, 1 Discal-, 1 Medianrand- und 1 Seitenrandborste. Endtergit insgesamt mit 8 Randborsten und 2 Discalborstenpaaren, ohne Tastborsten. Sternitborsten einfach zugespitzt, nur die des Endsternits zum Teil fein gezähnt, auf dem 10. und 11. Sternit je ein submedianes bezw. sublaterales Borstenpaar etwas länger. Chelicerenstamm mit 5 Borsten ( $S B$ vorhanden). Galea beim Männchen einfach pfriemenförmig, beim Weibchen mit apikalen Krallenästchen. Trochanter, Femur und Tibia der Palpen, besonders beim Männchen, hell bräunlichgelb, die Schere dunkelbraun. Trochanterhöcker ziemlich flach. Femur gut, aber nicht abrupt gestielt, 4 mal (†) bis

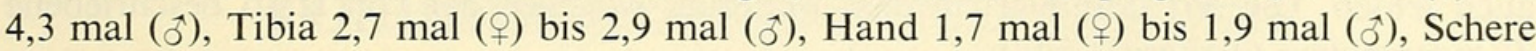
mit Stiel 3,1 mal (o) bis 3,3 mal ( $(\hat{)})$, ohne Stiel 2,9 bis 3,1 mal länger als breit. Finger etwas länger als die Hand ohne Stiel, die Tasthaare ist und it in der Regel nahe der Fingermitte ziemlich dicht beisammen stehend, selten etwas weiter getrennt. Vordertarsus des Männchens schlank, leicht spindelförmig, ohne Apikaldorn, die Klauen asymmetrisch, die innere mit einem dorsalen Subapikalzähnchen. Hintercoxen des Männchens mit einem Lateraldorn. Subterminalborste aller Beinpaare gezähnt. Beim Weibchen die Klauen aller Beinpaare, beim Männchen diejenigen des 2. bis 4. Paares apikal in der Regel, und zwar meist asymmetrisch gespalten, wobei die Länge der 2. Spitze sehr variabel ist; sie erscheint oft nur als kurzer Nebenzahn, der bei Exemplaren von der Zentralkordillere auch gänzlich geschwunden sein kann, so daß dann die Klauen einfach sind. Hintertarsus subapikal mit einer Tastborste, die etwa $2 \frac{1}{2} \mathrm{mal}$ so lang ist wie die Breite des Gliedes. - Körper-L. ô 1,6-1,7 mm, o 1,6-2 mm; Carapax-L. ơ $0,66 \mathrm{~mm}, \uparrow 0,72 \mathrm{~mm}, \mathrm{~B}$. o $0,54-0,60 \mathrm{~mm}, \uparrow 0,60 \mathrm{~mm}$; Palpen: ơ Femur-L. $0,70 \mathrm{~mm}$, B. $0,16 \mathrm{~mm}$, Tibia-L. 0,58-0,60 mm, B. 0,19-0,21 mm, Hand-L. 0,58$0,60 \mathrm{~mm}$, B. $0,30-0,32 \mathrm{~mm}$, Finger-L. $0,53-0,55 \mathrm{~mm}$; f Femur-L. 0,67 mm, B. $0,17 \mathrm{~mm}$, Tibia-L. $0,60 \mathrm{~mm}$, B. $0,22 \mathrm{~mm}$, Hand-L. $0,60 \mathrm{~mm}$, B. $0,35 \mathrm{~mm}$, Finger-L. $0,50 \mathrm{~mm}$.

Obwohl der männliche Vordertarsus keinen Apikaldorn besitzt, stelle ich diese Art zur Gattung Parachelifer, da die übrigen, m.E. wesentlichen Merkmale, insbesondere die normalerweise gespaltenen Klauen, die gezähnte Subterminalborste, die subdistale Tastborste der Hintertarsen und vor allem das charakteristische Dorsalzähnchen der Innenklaue der männlichen Vorderbeine für diese Genuszugehörigkeit sprechen. Der Apikaldorn der männlichen Vordertarsen ist erfahrungsgemäß auch bei Hysterochelifer sehr variabel und kann dort, wo er normalerweise vorhanden ist, bei ganzen Populationen auch mehr oder weniger stark reduziert sein oder sogar gänzlich fehlen. Er kann also nicht als Genusmerkmal herangezogen werden. Nach Hoff (1946) ist bei Idiochelifer die Subterminalborste gezähnt, weshalb ich dieses Genus in die Synonymie von Parachelifer stelle.

Parachelifer dominicanus zeichnet sich durch seine geringe Größe und die in der Regel nahe der Fingermitte beisammen stehenden Tasthaare ist und it des festen Palpenfingers aus.

\section{Cubachelifer strator Hoff, 1946}

1 i vom Valle Nuevo, Cord. centr., 2400 m, 16.VIII.1972, stelle ich mit Vorbehalt zu dieser Art. Die Subterminalborste ist gezähnt, die Klauen sind einfach. Der Hintertarsus trägt subapikal eine lange Tastborste. 
Tyrannochelifer floridanus (Banks, 1891)

129 oิ, 117 क, 23 Deuto- und Tritonymphen von folgenden Fundorten: Boca Chica, $10 \mathrm{~m}$, 5.-13.XII.1970, 22.V.-20.XI.1971, 25.III.-3.VI.1972; St. Domingo, $30 \mathrm{~m}$, 24.XII.1970 und 13.III.1971; Bani, $65 \mathrm{~m}$, 31.I.-24.X.1971, 23.I.-4.XI.1972, 3.II.1973, 19.I.-2.III.1974; Limon de Juna, 8.VI.1971; Jarabacoa, Cord. centr., 20.XII.1970, 22.III.1973 und 22.IV.1974; Cazabita, Cord. centr., 28.XI.1971 und 23.XII.1972; Colonia, Cord. centr., 1000 m. 10.VI.1972.

Der Tarsaldorn des männlichen Vordertarsus ist meist gut entwickelt, kann aber auch fehlen. Die Art dominiert in dem Material, wogegen der von Haiti beschriebene T. macropalpus (Tullgren) fehlt.

\section{LITERATUR}

Hoff, C. C. 1946. New pseudoscorpions, chiefly neotropical, of the suborder Monosphyronida. - Am. Mus. Novit. 1318: 1-32.

Hoff, C. C. 1959. The pseudoscorpions of Jamaica. Part I. The genus Tyrannochthonius. - Bull. Inst. Jamaica, sc. Ser. No. 10, pt. I: 1-39.

Anschrift des Verfassers:

Naturhistorisches Museum

Zoologische Abteilung

Postfach 417

Burgring 7

A-1014 Wien

Osterreich 


\section{$2 \mathrm{BHL}$ Biodiversity Heritage Library}

1976. "Pseudoscorpione von der Dominicanischen Republik (Inset Haiti)." Revue suisse de zoologie 83, 45-58. https://doi.org/10.5962/bhl.part.91433.

View This Item Online: https://www.biodiversitylibrary.org/item/129622

DOI: https://doi.org/10.5962/bhl.part.91433

Permalink: https://www.biodiversitylibrary.org/partpdf/91433

\section{Holding Institution}

Smithsonian Libraries

\section{Sponsored by}

Biodiversity Heritage Library

\section{Copyright \& Reuse}

Copyright Status: In Copyright. Digitized with the permission of the rights holder.

Rights Holder: Muséum d'histoire naturelle - Ville de Genève License: http://creativecommons.org/licenses/by-nc-sa/3.0/

Rights: https://www.biodiversitylibrary.org/permissions/

This document was created from content at the Biodiversity Heritage Library, the world's largest open access digital library for biodiversity literature and archives. Visit BHL at https://www.biodiversitylibrary.org. 